\title{
Cytokine-induced killer cells induce apoptosis and inhibit the Akt/nuclear factor-кB signaling pathway in cisplatin-resistant human glioma U87MG cells
}

\author{
YUNPENG CUI $^{1 *}$, FENG YANG $^{2 *}$ and $\mathrm{LU} \mathrm{HE}^{3}$ \\ ${ }^{1}$ Department of Clinical Laboratory, Tianjin Huanhu Hospital, Tianjin 300060; ${ }^{2}$ Department of Neurosurgery, \\ The Sixth People's Hospital of Chongqing City, Chongqing 400060; ${ }^{3}$ Department of Anatomy and Histology, \\ School of Basic Medical Sciences, Tianjin Medical University, Tianjin 300070, P.R. China
}

Received October 23, 2014; Accepted July 22, 2015

DOI: $10.3892 / \mathrm{mmr} .2015 .4236$

\begin{abstract}
Despite advances in the development of treatment methods, glioma remains among the cancer types with a high rate of mortality. Therefore, significant efforts are made to develop novel strategies for the treatment of glioma. Ineffective, long-term cancer chemotherapy can lead to multidrug resistance (MDR), which is one of the most common reasons for the failure of chemotherapy. The present study investigated the effects of cytokine-induced killer cells (CIK) on reversing MDR in cisplatin-resistant U87MG cells (U87MG/DDP). Mononuclear cells were isolated from the peripheral blood of healthy individuals and cultured in vitro in the presence of a combination of cytokines to generate CIK for the treatment of U87MG/DDP. An MTS assay, flow cytometric analysis of apoptosis, ELISA, western blotting and reverse transcription quantitative polymerase chain reaction were used to investigate the MDR-reversing effects of CIK as well as the underlying mechanisms. The results showed that cisplatin sensitivity and the apoptotic rate following cisplatin treatment were increased, P-glycoprotein expression was decreased and the intracellular rhodamine-123 content was increased in U87MG/DDP co-cultured with CIK. In addition, the present study also identified increased mRNA and protein expression levels of MDR gene 1 (MDR1), MDR-associated protein 1 (MRP1), B-cell lymphoma 2, Survivin and glutathione S-transferase- $\pi$, while the phosphorylation of AKT and the transcriptional activity of nuclear factor- $\kappa \mathrm{B}$ in CIK co-cultured U87MG/DDP was decreased. These results indicated that pre-treatment with CIK reversed the MDR of U87MG/DDP, and that CIK-induced
\end{abstract}

Correspondence to: Dr Lu He, Department of Anatomy and Histology, School of Basic Medical Sciences, Tianjin Medical University, 22 Qixiangtai Road, Tianjin 300070, P.R. China E-mail: helu@tmu.edu.cn

${ }^{*}$ Contributed equally

Key words: cytokine-induced killer, glioma, cisplatin resistance apoptosis of U87MG/DDP was associated with the inhibition of Akt/NF-kB. These findings suggested that treatment with CIK may be an effective method to enhance the sensitivity of patients with glioma to chemotherapy.

\section{Introduction}

Chemotherapy is one of the most important methods for the clinical treatment of glioma. However, drug resistance of glioma cells is gradually induced with the long-term application of chemotherapeutic drugs, eventually resulting in treatment failure. Multidrug resistance (MDR) is the main form of drug resistance in glioma $(1,2)$. The MDR gene mediates this process and constitutes the classic pathway of drug resistance. The MDR gene has now a the major prognostic factor for patients with glioma. Therefore, the reversal of drug resistance is an urgent issue to be addressed in the treatment of glioma and may improve treatment outcomes.

With the advances in immunology, molecular biology and oncology, adoptive immunotherapy has attracted large amounts of attention as an important treatment method for tumors following chemotherapy $(3,4)$. Phytohemagglutinin (PHA) as a polyclonal activator of cells can induce the transformation of immature lymphocytes into lymphoblasts, followed by proliferation, release of lymphokines and enhanced phagocytosis of macrophages (5). Conventional cytokine-induced killer cells (CIK) are a type of immunological effector cells with potent killing ability. They are derived from human peripheral blood mononuclear cells following stimulation with anti-CD3 monoclonal antibody, interleukin (IL)-1, IL-2 and interferon (IFN)- $\gamma$ under in vitro conditions, simulating the in vivo physiological environment $(6,7)$.

In the present study, CIK were generated by stimulating peripheral blood mononuclear cells with PHA, IL-2 and IFN- $\gamma$. The potency of these CIK against a cisplatin-resistant U87MG glioma cell line (U87MG/DDP) was studied and the mechanisms of action were investigated. CIK were cultured in order to provide not only sufficient quantities of highly efficient killer cells for future implementation of tumor biotherapy, but also a novel method for the adoptive immunotherapy of glioma. 


\section{Materials and methods}

Preparation and culturing of cells. Mononuclear cells were isolated from peripheral blood obtained from healthy volunteers using density-gradient centrifugation (speed, $800 \mathrm{x} \mathrm{g}$; duration, $20 \mathrm{~min}$ ) with lymphocyte separation medium (density, $1.077 \pm 0.002$ ). The mononuclear cells were re-suspended in cell culture medium (Beyotime Institute of Biotechnology, Shanghai, China) to a density of $4.0 \times 10^{6} / \mathrm{ml}$. On day 0, the cells were co-stimulated with PHA $(25 \mu \mathrm{g} / \mathrm{ml}$; Sigma-Aldrich, St. Louis, MO, USA) and IFN- $\gamma(300 \mathrm{U} / \mathrm{ml}$; R\&D Systems, Inc., Minneapolis, MN, USA) for culture in an incubator at $37^{\circ} \mathrm{C}$ with an atmosphere containing $5 \% \mathrm{CO}_{2}$ for 24 h. On day 2, IL-2 (1,000 U/ml; R\&D Systems, Inc.) was added. The medium was replaced with medium containing IL-2 $(1,000 \mathrm{U} / \mathrm{ml})$ once every three days until the effector cells were harvested on day 20. The U87MG cells and U87MG/DDP cell lines were obtained from Tongpai Biological Technology Co., Ltd. (Shanghai, China).

ELISA assay. A total of $5 \times 10^{5} \mathrm{CIK}$ were harvested and cytokine secretion by the CIK was detected by ELISA (R\&D Systems). For this, $3 \times 10^{5}$ cells were seeded into wells of a six-well microplate and incubated overnight. Medium without fetal calf serum was added and the secretion of IFN- $\gamma$, IL-2, IL- 6 and IL- 12 was measured $72 \mathrm{~h}$ later following the manufacturer's instructions.

MTS assay. Cells in the logarithmic growth phase were seeded in a 96-well microplate at $100 \mu \mathrm{l} / \mathrm{well}\left(5 \times 10^{4} \mathrm{cells} / \mathrm{ml}\right)$ and cultured overnight to allow for cell attachment. Subsequently, $0,0.1,0.5,1,5,10,50$ and $100 \mu \mathrm{M}$ cisplatin (Sigma-Aldrich) was added. The effector (CIK)/target (U87MG/DDP) ratio (E/T ratio) was 10:1 and 20:1. After continuous culture for $72 \mathrm{~h}$, the medium was removed. MTS (Promega Corporation, Madison, WI, USA) was added in accordance with the manufacturer's instructions and incubation was continued for $4 \mathrm{~h}$. Finally, the optical density (OD) at $490 \mathrm{~nm}$ wavelength was detected using a microplate reader (Multiskan ${ }^{\circledR}$ Spectrum; Thermo Scientific, Rockford, IL, USA). The inhibition rate of cisplatin on the cells was calculated as follows: Inhibition rate $=\left(1-\mathrm{OD}_{\text {experimental }}\right.$ group $\left./ \mathrm{OD}_{\text {control group }}\right) \mathrm{x} 100 \%$. Displaying the cisplatin concentration on the abscissa and the inhibition rate on the ordinate, the curve was plotted and fitted to obtain the $\mathrm{IC}_{50}$ value. The reversal fold (RF) was calculated as $\mathrm{RF}=\mathrm{IC}_{50}\left(\mathrm{CIK}\right.$-treated group)/IC $\mathrm{C}_{50}$ (control group).

The cells were divided into the following treatment groups: Parental group, U87MG cells; Group I, U87MG/DDP; Group II, U87MG/DDP with 10:1 CIK; Group III, U87MG/DDP with 20:1 CIK. The following assays were performed on 10:1 and 20:1 mixtures of glioma and CIK cells.

Flow cytometry. After treatment with cisplatin and CIK for $72 \mathrm{~h}$, the tumor cells were collected and stained with fluorescein isothiocyanate (FITC)-conjugated Annexin V/propidium iodide (PI) away from light for $15 \mathrm{~min}$. Apoptosis was then detected by flow cytometry (BD FACSAria ${ }^{\mathrm{TM}}$; BD Biosciences, Franklin Lakes, NJ, USA). The apoptotic rate was calculated using FACSDiva software version 8.0 (BD Biosciences).

After treatment with CIK for $72 \mathrm{~h}$, the tumor cells were collected and cultured with phycoerythrin-P-glycoprotein
(P-gp) or rhodamine (Rh)-123 antibody away from light for 30 min. P-gp and Rh-123 expression in these cells was detected using flow cytometry and the P-gp- or Rh-123-positive rate was calculated using FACSDiva software.

For cell cycle analysis, cells were treatment with CIK for $72 \mathrm{~h}$ and the tumor cells were collected and incubated with PI away from light for $30 \mathrm{~min}$. The cell cycle was determined by flow cytometry and calculated using ModFit3.0 software (Verity Software House, Topsham, ME, USA).

Western blot analysis. After treatment with CIK for $72 \mathrm{~h}$, the tumor cells were collected and lysed using lysis buffer (Beyotime Institute of Biotechnology) to extract the total proteins. The concentration was measured using a BCA Protein assay kit (Beyotime Institute of Biotechnology). Proteins $(100 \mu \mathrm{g})$ were separated using 12\% SDS-PAGE and transferred onto a polyvinylidene difluoride membrane. After blocking in $5 \%$ skimmed milk powder at room temperature for $1 \mathrm{~h}$, the membrane was incubated with MDR1 [cat. no. sc-55510; mouse immunoglobulin (Ig)G], MDR-associated protein (MRP)1 (cat. no. sc-365635; mouse IgG1), B-cell lymphoma 2 (Bcl-2; cat.no. sc-7382; mouse IgG1), survivin (cat.no. sc-374616; mouse IgG1), glutathione S-transferase (GST)- $\pi$ (cat. no. sc-374171; mouse IgG1), nuclear factor- $\mathrm{kB}$ (cat. no. sc-292436; rabbit IgG), total-AKT (cat. no. sc-5298; mouse IgG1) and phosphorylated (p)-Akt (cat. no. sc-293125; mouse IgG1) primary antibodies at $4^{\circ} \mathrm{C}$ overnight (dilution, 1:2,000; all obtained from Santa Cruz Biotechnology, Inc., Dallas, TX, USA). $\beta$-actin (cat. no. sc-8432; mouse IgG1) served as an internal control (dilution, 1:5,000; Santa Cruz Biotechnology, Inc.). After washing the primary antibodies off with $10 \%$ phosphate-buffered saline (three washes), the membrane was incubated with horseradish peroxidase-conjugated goat anti-rabbit immunoglobulin $\mathrm{G}$ at room temperature for $1 \mathrm{~h}$. After washing, the immunoreactive bands were developed by enhanced chemiluminescence (EMD Millipore, Billerica, MA, USA) and the film was obtained from SiDaTe (Suzhou, China). The relative expression was represented as the ratio of target protein and $\beta$-actin bands.

Reverse-transcription quantitative polymerase chain reaction (RT-qPCR) assay. After treatment with CIK for $72 \mathrm{~h}$, the tumor cells were collected to extract the total RNA from each group using the TRIzol reagent (Invitrogen Life Technologies, Carlsbad, CA, USA) according to the manufacturer's instructions. The cDNA was obtained through reverse transcription using a real-time PCR kit (Tiangen Biotech Co., Ltd., Beijing, China) and an ABI7500 Real-Time PCR system (Applied Biosystems Life Technologies, Foster City, CA, USA). After denaturation at $94^{\circ} \mathrm{C}$ for $5 \mathrm{~min}, 35$ cycles of amplification were performed under the following conditions: $95^{\circ} \mathrm{C}$ for $15 \mathrm{sec}$, $65^{\circ} \mathrm{C}$ for $40 \mathrm{sec}$ and $72^{\circ} \mathrm{C}$ for $90 \mathrm{sec}$; followed by extension at $72^{\circ} \mathrm{C}$ for $5 \mathrm{~min}$ after these cycles. The oligonucleotide sequences (Sangon Biotech Co., Ltd., Shanghai, China) were as follows: MDR1 forward, 5'-AAAAAGATCAACTCGTAC CACTC-3' and reverse, 5'-GCACAAAATACACCAACAA-3'; MRP1 forward, 5'-GTGGAATTCCGGAACTAC-3' and reverse, 5'-CGGAGGTCGTGCAGGCCG-3'; GST- $\pi$ forward, 5'-CTGGAAGGAGGAGGTGGTG-3' and reverse, 5'-GAC GCAGGATGGTATTGGAC-3'; Survivin forward, 5'-CGA GGCTGGCTTCATCCACT-3' and reverse 5'-ACGGCGCAC 


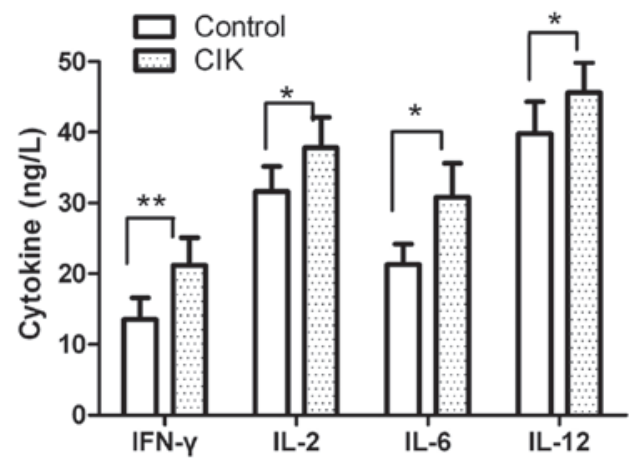

Figure 1. Cytokine secretion levels of INF- $\gamma$, IL-2, IL-6, IL-10 and IL-12. The cytokine secretion by CIK after 24 and $72 \mathrm{~h}$ was analyzed by ELISA. Each experiment was performed three times and the control group was formed of unstimulated mononuclear blood lymphocytes. Values are expressed as the mean \pm standard deviation. ${ }^{*} \mathrm{P}<0.05 ;{ }^{* *} \mathrm{P}<0.01$. IFN $-\gamma$, interferon- $\gamma$; IL, interleukin; CIK, cytokine-induced killer cells.

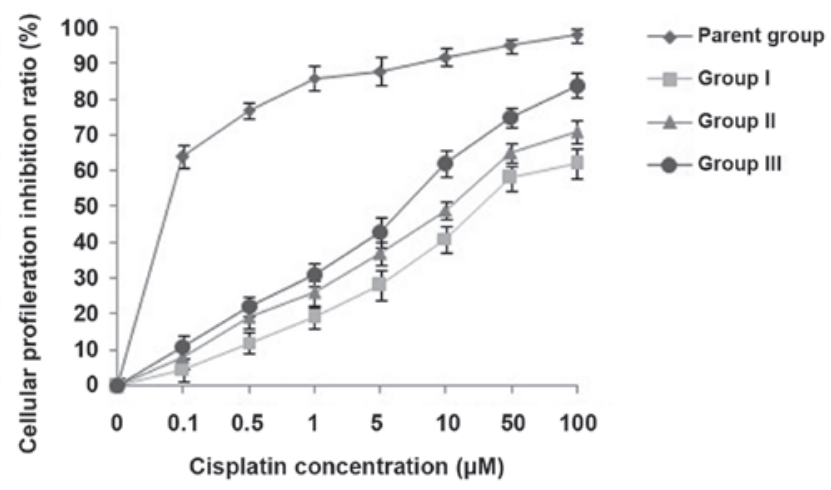

Figure 2. Effects of CIK on MDR reversal in U87MG/DDP cells. The MDR reversal effect of CIK cells on the U87MG/DDP and U87MG cells was assessed using an MTS assay. Values are expressed as the mean \pm standard deviation $(n=5)$. $\mathrm{P}<0.05$, groups I, II and III vs. the parent group; groups I and II vs. group III; group I vs. group II. Groups: Parent group, native U87MG cells; I, U87MG/DDP; II, U87MG/DDP with 10:1 CIK; III, U87MG/DDP with 20:1 CIK. MDR, multidrug-resistance; CIK, cytokine-induced killer cells; U87MG/DDPs, cisplatin-resistant U87MG cell line.

A

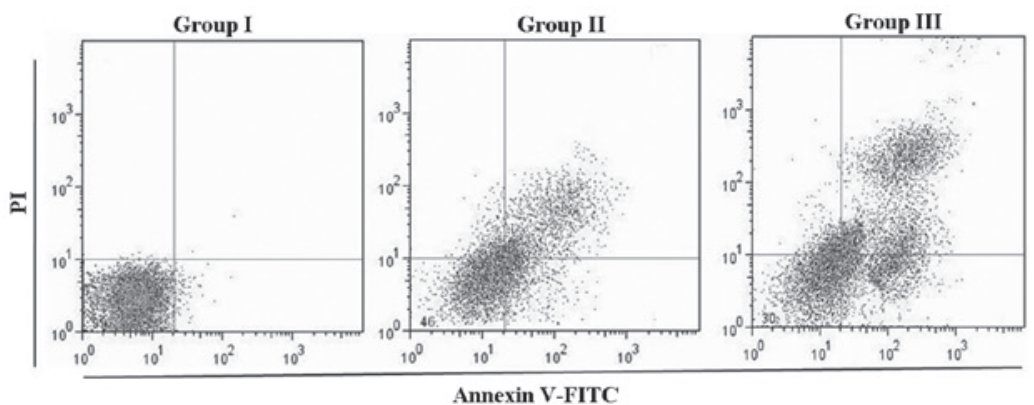

B

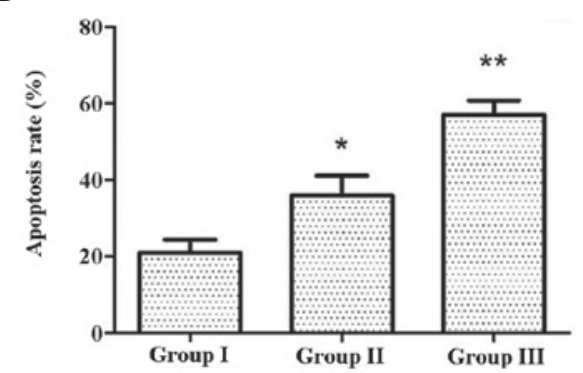

Figure 3. Effect of CIK on apoptosis induction in U87MG/DDP cells. (A) Apoptosis of U87MG/DDP cells incubated with CIK was assessed using flow cytometric analysis. (B) Quantified apoptotic rates. Values are expressed as the mean \pm standard deviation $(\mathrm{n}=3)$. " $\mathrm{P}<0.05$; ${ }^{* *} \mathrm{P}<0.01$ vs. Group I. Groups: I, U87MG/DDP; II, U87MG/DDP with 10:1 CIK; III, U87MG/DDP with 20:1 CIK. CIK, cytokine-induced killer cells; U87MG/DDP, cisplatin-resistant U87MG cell line; FITC, fluorescein isothiocyanate; PI, propidium iodide. ${ }^{*} \mathrm{P}<0.05$ and ${ }^{* *} \mathrm{P}<0.01$ vs. Group I.

TTTCTTCGCA-3'; Bcl-2 forward, 5'-GGCTGGGATGCC TTTGTG-3' and reverse, 5'-GCCAGGAGAAATCAAACA GAGG-3'; GAPDH forward, 5'-GGAGCGAGATCCCTC CAAAAT-3' and reverse, 5'-GGCTGTTGTCATACTTCT CATGG-3'. The PCR products were quantified using the $2^{-\Delta \Delta C t}$ method.

Statistical analysis. Values are expressed as the mean \pm standard deviation. SPSS 21.0 software (International Business
Machines, Armonk, NY, USA) was used for analysis. One-way analysis of variance was used for comparison and $\mathrm{P}<0.05$ indicated a statistically significant difference.

\section{Results}

Cell cytokine secretion by CIK. The cell cytokine secretion of CIK was detected using an ELISA assay. The CIK mainly produced IFN- $\gamma$, IL-2, IL- 6 and IL-12 at the end of the 
A

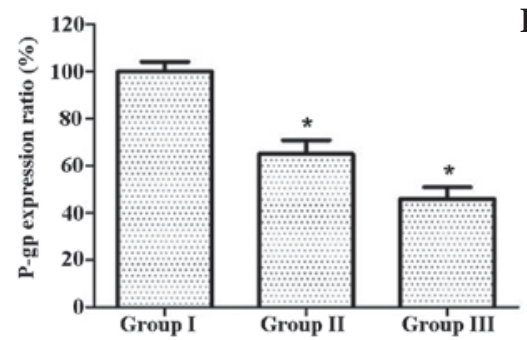

B

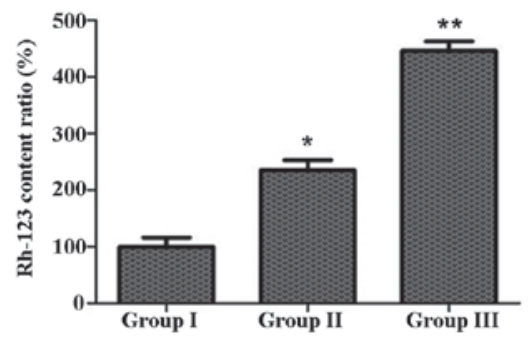

Figure 4. Effects of CIK on intracellular Rh-123 content and P-gp expression in U87MG/DDP cells. (A) Effects of CIK on P-gp expression in U87MG/DDP cells. (B) Effects of CIK on the intracellular Rh-123 content of U87MG/DDP cells. Values are expressed as the mean \pm standard deviation ( $\mathrm{n}=3$ ). ${ }^{*} \mathrm{P}<0.05$ and ${ }^{* *} \mathrm{P}<0.01$ vs. Group I. Groups: I, U87MG/DDP; II, U87MG/DDP with 10:1 CIK; III, U87MG/DDP with 20:1 CIK. CIK, cytokine-induced killer cells; U87MG/DDP, cisplatin-resistant U87MG cell line; P-gp, P-glycoprotein; Rh, rhodamine.

A

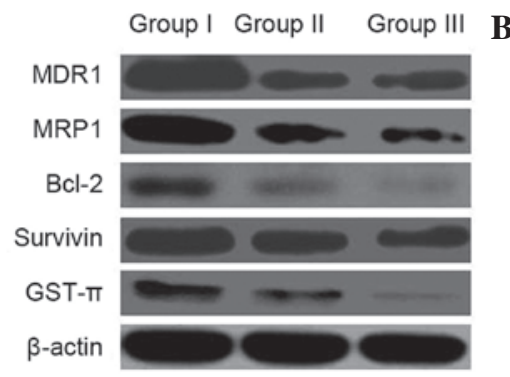

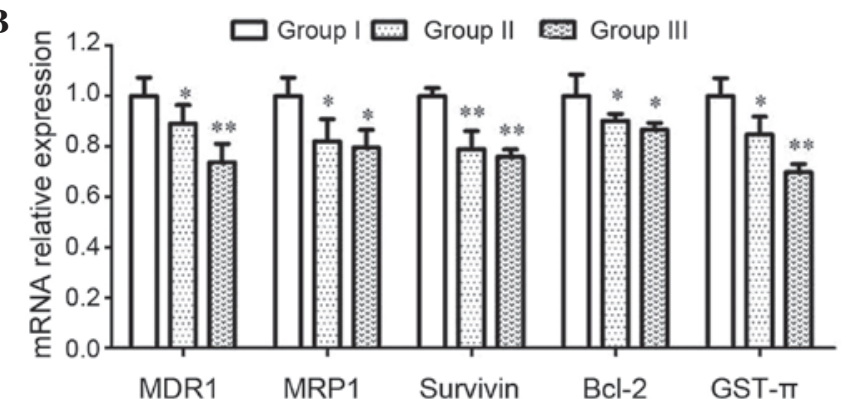

Figure 5. Effects of CIK on MDR-associated proteins and genes in U87MG/DDP cells. (A) U87MG/DDP cells were treated for $72 \mathrm{~h}$, and the protein expression of MDR1, MRP1, Bcl-2, Survivin and GST- $\pi$ was assessed using western blotting. (B) mRNA levels of MDR1, MRP1, Bcl-2, Survivin and GST- $\pi$ were assessed by reverse transcription-quantitative polymerase chain reaction assays in cells. GAPDH served as an internal reference. Values are expressed as the mean \pm standard deviation $(\mathrm{n}=5)$. " $\mathrm{P}<0.05$ and ${ }^{* *} \mathrm{P}<0.01$. Groups: I, U87MG/DDP; II, U87MG/DDP with 10:1 CIK; III, U87MG/DDP with 20:1 CIK. CIK, cytokine-induced killer cells; U87MG/DDP, cisplatin-resistant U87MG cell line; MDR, multidrug-resistance; MRP, MDR-associated protein; Bcl-2, B-cell lymphoma 2; GST, glutathione S-transferase.

culturing period. Cytokine secretion levels in each group were assessed prior to and after inductive cytokine treatments. As shown in Fig. 1, the levels of IFN- $\gamma$, IL-2, IL-6 and IL-12 were slightly enhanced following culture for $72 \mathrm{~h}$.

Effects of CIK cells on MDR reversal in U87MG/DDP cells. The viability of U87MG/DDP cells treated with cisplatin at various concentrations $(0,0.1,0.5,1,5,10,50$ and $100 \mu \mathrm{M})$ for $72 \mathrm{~h}$ was assessed and the effects of co-incubation with CIK on MDR reversal in U87MG/DDP were evaluated using an MTS assay. As shown in Fig. 2, the cytotoxicity of cisplatin to group-II cells was higher than that to group-I cells, while that to group-III cells was even higher, indicating an MDR-reversing effect of CIK on U87MG/DDP.

CIK induce apoptosis in U87MG/DDP. To investigate the effects of CIK on apoptosis, Annexin V-FITC/PI double staining was employed. As shown in Fig. 3, the apoptotic rate in group II was higher than that in group I, while that in group III was even higher. This indicated that co-culture with CIK increased the apoptotic rate of U87MG/DDP.

CIK induce Rh-123 production and reduce $P$-gp expression in $U 87 M G / D D P$. The intracellular Rh-123 content and expression of P-gp were analyzed by flow cytometry. As shown in Fig. 4, the intracellular Rh-123 content in group II was increased compared with that in group I, and the expression of P-gp in group II was lower than that in group I; these effects

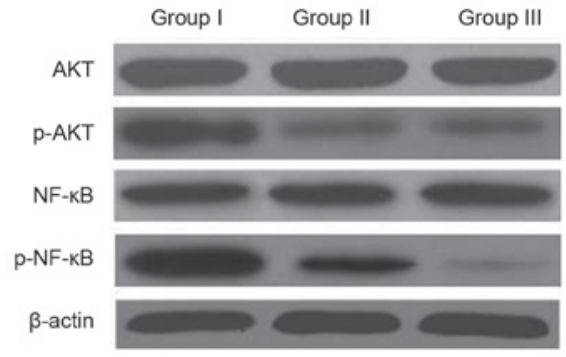

Figure 6. Effects of CIK on the AKT/NF- $\mathrm{B}$ signaling pathway in U87MG/DDP cells. The protein expression of total-AKT, p-AKT and NF-kB was assessed by western blot analysis. Groups: I, U87MG/DDP; II, U87MG/DDP with 10:1 CIK; III, U87MG/DDP with 20:1 CIK. CIK, cytokine-induced killer cells; U87MG/DDP, cisplatin-resistant U87MG cell line; $\mathrm{p}$, phosphorylated; NF, nuclear factor.

were even more marked in group III. These results indicated that co-incubation with CIK increased the intracellular Rh-123 content, while having an inhibitory effect on P-gp expression in U87MG/DDP cells.

CIK reduces MDR-associated gene expression in $U 87 M G / D D P$. To further study the mechanism of the MDR reversal by CIK, the expression of MDR-associated proteins and genes (MDR1, MRP1, Bcl-2, Survivin and GST- $\pi$ ) was analyzed by western blot and RT-qPCR analyses. As shown in Fig. 5, the protein and mRNA expression of these genes in group II was lower than that in group I, while it was even 
lower in group III, indicating an inhibitory effect of CIK on the expression of MDR-associated genes in U87MG/DDP.

The NF- $\kappa B / A K T$ signaling pathway is involved in the MDR reversal of $C I K$. It has been reported that activation of $N F-\kappa B$ followed by Akt phosphorylation has a role in the regulation of cell survival, apoptosis and drug resistance $(8,9)$. The present study therefore investigated whether the intracellular AKT/NF- $\mathrm{B}$ signaling pathway was involved in the effects of $\mathrm{CIK}$ on MDR. The expression of $\mathrm{NF}-\kappa \mathrm{B}$ and AKT was assessed by western blot analysis. As shown in Fig. 6, co-incubation with CIK decreased the phosphorylation of AKT, but did not affect total-Akt expression. Furthermore, the expression levels of $\mathrm{NF}-\kappa \mathrm{B}$ were decreased in U87MG/DDP co-incubated with CIK. These results suggested that CIK induced apoptosis and MDR reversal by inactivation of $N F-\kappa B$ via the $A k t / N F-\kappa B$ pathway.

\section{Discussion}

Due to the insidious onset of glioma and high degree of malignancy, most patients are already in intermediate-to-advanced stage at the time of diagnosis, which is characterized by a low rate of applicability of resection and frequently occurring post-operative local recurrence and metastasis. In addition, the poor immune function in patients with intermediate-to-advanced stage glioma further results in poor clinical efficacy $(10,11)$. Accordingly, it is of high importance to identify novel treatment protocols, improve the quality of life in patients with intermediate-to-advanced stage glioma and to increase their survival rate. In recent years, biotherapy has attracted increasing attention as a treatment option for glioma $(12,13)$.

Based on the enormous potential of immune cells in tumor treatment, biotherapy has become a novel and increasingly emphasized means of comprehensive tumor treatment. CIK have the potent anti-tumor activity of $\mathrm{T}$ cells as well as the non-major histocompatibility complex-restricted cytotoxicity of natural killer cells with a high proliferation rate and fewer toxic/side effects (14). As experimental and clinical studies have confirmed the efficacy of CIK on malignant tumors, CIK have rapidly become a focus of tumor immunotherapy research. A significant difference in cisplatin sensitivity with 10:1 and 20:1 mixtures of glioma and CIK cells was identified.

Given the fact that the mechanisms of the drug resistance of glioma cells are closely associated with MDR genes, effective downregulation of these genes is the primary approach to reverse drug resistance (15-17). MDR1 is expressed in normal tissue and cell types. P-glycoprotein (P-gp), encoded by MDR1, is embedded in the cell membrane surface to form an efflux pump. P-gp can also effect the secretion and transport of lipids and exert additional functions, among which antiport predominates $(18,19)$. The physiological significance of P-gp is to prevent cytotoxicity resulting from intracellular accumulation of endogenous or exogenous lipid-soluble substances, including certain cellular metabolites, toxicants and other substances in order to maintain a relatively stable intracellular milieu. Certain lipid-soluble and naturally derived drugs can induce P-gp expression to easily cause the development of multi-drug resistance. $\mathrm{Rh}-123$ is a classic substrate for the examination of $\mathrm{P}$-gp activity; the in vivo elimination of Rh-123 is only correlated with P-gp activity and can therefore be used to determine intracellular drug concentrations (20). CIK can effectively downregulate the expression of intracellular MDR1 and P-gp, while upregulating Rh-123 (21).

MRP1, a member of the adenosine triphosphate (ATP) binding cassette transporter protein super-family, can upregulate the expression of ATP-dependent GST- $\pi$ to excrete conjugated anions from the cells and have a role in the clearance of exogenous toxins $(22,23)$. In the present study, CIK effectively downregulated the expression of intracellular MRP1 and GST- $\pi$, which may represent one of the mechanisms for the reversal of tumor drug resistance. Bcl-2 and Survivin are two common anti-apoptotic proteins, which are significantly upregulated in drug-resistant tumor cells compared with non-resistant tumor cells. The present study confirmed that co-culture with CIK downregulated Bcl-2 and Survivin levels and increased apoptosis in U87MG/DPP. Furthermore, co-culture with CIK downregulated the expression of NF- $\kappa \mathrm{B}$ and p-Akt in U87MG/DPP, suggesting that the activity of this signaling pathway was decreased and that NF- $\kappa \mathrm{B}$ and $\mathrm{p}-\mathrm{Akt}$ signaling pathways were involved in CIK-mediated reversal of drug resistance.

The drug resistance of glioma is the underlying cause of the limited efficacy of clinical treatments. Identifying effective reversal methods is therefore a contemporary issue to be addressed. The results of the present study showed that co-culture with CIK was able to reverse the resistance of glioma cells to the chemotherapeutic drug cisplatin in vitro. This enhancement of the sensitivity of glioma cells was mediated via the NF- $\kappa \mathrm{B}$ and p-Akt signaling pathways, the induction of apoptosis and the reduction of the expression of MDR-associated genes. Future clinical studies should continuously aim to discover and explore methods with enhanced effectiveness in order to provide novel approaches for the development of clinical treatments to overcome drug resistance. CIK as a novel therapeutic have been employed in the clinical treatment of tumors due to their high tumoricidal activity, low side effects and other characteristics. Given the promising application prospect of this method, it is necessary to perform more in-depth studies.

\section{Acknowledgements}

This study was supported by the National Natural Science Foundation of China (grant no. 81200957).

\section{References}

1. Fan QW and Weiss WA: Targeting the RTK-PI3K-mTOR axis in malignant glioma: Overcoming resistance. Curr Top Microbiol Immunol 347: 279-296, 2010.

2. Desjardins A, Rich JN, Quinn JA, Vredenburgh J, Gururangan S, Sathornsumetee S, Reardon DA, Friedman AH, Bigner DD and Friedman HS: Chemotherapy and novel therapeutic approaches in malignant glioma. Front Biosci 10: 2645-2668, 2005.

3. Curiel TJ: Immunotherapy: A useful strategy to help combat multidrug resistance. Drug Resist Updat 15: 106-113, 2012.

4. Ji J, Black KL and Yu JS: Glioma stem cell research for the development of immunotherapy. Neurosurg Clin N Am 21: $159-166,2010$ 
5. Tu W, Cheung PT and Lau YL: Insulin-like growth factor 1 promotes cord blood $\mathrm{T}$ cell maturation and inhibits its spontaneous and phytohemagglutinin-induced apoptosis through different mechanisms. J Immunol 165: 1331-1336, 2000

6. Kim JS, Chung IS, Lim SH, Park Y, Park MJ, Kim JY, Kim YG, Hong JT, Kim Y and Han SB: Preclinical and clinical studies on cytokine-induced killer cells for the treatment of renal cell carcinoma. Arch Pharm Res 37: 559-566, 2014.

7. Rettinger E, Kreyenberg H, Merker M, Kuci S, Willasch A, Bug G, Ullrich E, Wels WS, Bonig H, Klingebiel T and Bader P: Immunomagnetic selection or irradiation eliminates alloreactive cells but also reduces anti-tumor potential of cytokine-induced killer cells: Implications for unmanipulated cytokine-induced killer cell infusion. Cytotherapy 16: 835-844, 2014.

8. Zhou W, Fu XQ, Zhang LL, Zhang J, Huang X, Lu XH, Shen L, Liu BN,Liu J,LuoHS, etal:The AKT1/NF-kappaB/Notch1/PTEN axis has an important role in chemoresistance of gastric cancer cells. Cell Death Dis 4: e847, 2013.

9. Wang Y, Li CF, Pan LM and Gao ZL: 7,8-Dihydroxycoumarin inhibits A549 human lung adenocarcinoma cell proliferation by inducing apoptosis via suppression of Akt/NF- $\kappa \mathrm{B}$ signaling. Exp Ther Med 5: 1770-1774, 2013.

10. Piil K, Juhler M, Jakobsen J and Jarden M: Controlled rehabilitative and supportive care intervention trials in patients with high-grade gliomas and their caregivers: A systematic review. BMJ Support Palliat Care pii: bmjspcare-2013-000593, 2014.

11. Dirven L, Aaronson NK, Heimans JJ and Taphoorn MJ: Health-related quality of life in high-grade glioma patients. Chin J Cancer 33: 40-45, 2014

12. Melin B and Jenkins R: Genetics in glioma: Lessons learned from genome-wide association studies. Curr Opin Neurol 26: 688-692, 2013

13. Li W, Holsinger RM, Kruse CA, Flügel A and Graeber MB: The potential for genetically altered microglia to influence glioma treatment. CNS Neurol Disord Drug Targets 12: 750-762, 2013.

14. Zhang J, Zhu L, Zhang Q, He X, Yin Y, Gu Y, Guo R, Lu K Liu L, Liu P and Shu Y: Effects of cytokine-induced killer cell treatment in colorectal cancer patients: A retrospective study. Biomed Pharmacother 68: 715-720, 2014.
15. Queiroz RM, Takiya CM, Guimarães LP, Rocha Gda G, Alviano DS, Blank AF, Alviano CS and Gattass CR: Apoptosis-inducing effects of melissa officinalis L. essential oil in glioblastoma multiforme cells. Cancer Invest 32: 226-235, 2014.

16. Zhu Y, Liu XJ, Yang P, Zhao M, Lv LX, Zhang GD, Wang Q and Zhang L: Alkylglyceronephosphate synthase (AGPS) alters lipid signaling pathways and supports chemotherapy resistance of glioma and hepatic carcinoma cell lines. Asian Pac J Cancer Prev 15: 3219-3226, 2014.

17. Nakai E, Park K, Yawata T, Chihara T, Kumazawa A, Nakabayashi $\mathrm{H}$ and Shimizu K: Enhanced MDR1 expression and chemoresistance of cancer stem cells derived from glioblastoma. Cancer Invest 27: 901-908, 2009.

18. Goda K, Bacsó Z and Szabó G: Multidrug resistance through the spectacle of P-glycoprotein. Curr Cancer Drug Targets 9: 281-297, 2009.

19. Lage H: MDR1/P-glycoprotein (ABCB1) as target for RNA interference-mediated reversal of multidrug resistance. Curr Drug Targets 7: 813-821, 2006

20. Xu D, Tian W and Shen H: Curcumin prevents induced drug resistance: A novel function? Chin J Cancer Res 23: 218-223, 2011.

21. Wang L, Deng Q, Wang J, Bai X, Xiao X, Lv HR, Zhao MF and Liu PJ: Effect of CIK on multidrug-resistance reversal and increasing the sensitivity of ADR in K562/ADR cells. Oncol Lett 8: 1778-1782, 2014.

22. Peklak-Scott C, Smitherman PK, Townsend AJ and Morrow CS: Role of glutathione S-transferase P1-1 in the cellular detoxification of cisplatin. Mol Cancer Ther 7: 3247-3255, 2008.

23. Calatozzolo C, Gelati M, Ciusani E, Sciacca FL, Pollo B, Cajola L, Marras C, Silvani A, Vitellaro-Zuccarello L, Croci D, et al: Expression of drug resistance proteins Pgp, MRP1, MRP3, MRP5 and GST-pi in human glioma. J Neurooncol 74: 113-121, 2005. 